\title{
Corneal Graft Rejection after Descemet's Membrane Endothelial Keratoplasty with Peripheral Anterior Synechiae
}

\author{
Yukiko Miyoshi ${ }^{a} \quad$ Takashi Ono $^{a}$ Saori Seki ${ }^{a}$ Tetsuya Toyono ${ }^{a}$ \\ Kohdai Kitamoto $^{a}$ Takahiko Hayashi $^{b}$ Tomohiko Usuic ${ }^{c}$ Makoto Aihara ${ }^{a}$ \\ Takashi Miyai $^{a}$ \\ aDepartment of Ophthalmology, Graduate School of Medicine, The University of Tokyo, \\ Tokyo, Japan; 'bepartment of Ophthalmology, Yokohama Minami Kyousai Hospital, \\ Kanagawa, Japan; 'Department of Ophthalmology, International University of Health and \\ Welfare, Chiba, Japan
}

\author{
Keywords \\ Corneal transplantation - Descemet's membrane endothelial keratoplasty · Graft rejection · \\ Peripheral anterior synechiae
}

\begin{abstract}
Descemet's membrane endothelial keratoplasty (DMEK) for patients with corneal endothelial loss rarely results in graft rejection. Herein, we report a rare case of graft rejection following DMEK, in which peripheral anterior synechiae were observed postoperatively. A 66-year-old woman was referred to our hospital after complaints of decreased visual acuity of her right eye after laser iridotomy for primary angle closure 3 years earlier. Her right cornea had bullous keratopathy with mild cataract, and her best-corrected visual acuity (BCVA) was 20/40. After cataract surgery, DMEK was successfully performed, except for development of peripheral anterior synechiae at the temporal cornea. Her BCVA recovered to 20/20. However, when topical instillation was changed to $0.1 \%$ fluorometholone from $0.1 \%$ betamethasone once a day, corneal edema reappeared with hyperemia, mutton fat keratic precipitates (KPs), and cells in the anterior chamber. The BCVA worsened to 20/32. Graft rejection was diagnosed, and subconjunctival injection of dexamethasone was performed 3 times, once every few days, with $0.1 \%$ topical betamethasone instillation. Subsequently, the hyperemia, mutton fat KPs, and cells in the anterior chamber disappeared with a recovered BCVA of 20/20 after 2 weeks. Ten months
\end{abstract}

Yukiko Miyoshi and Takashi Ono contributed equally to this manuscript. 
after graft rejection, there was no recurrence of intraocular inflammation, and only topical betamethasone was administered twice daily. It is important to exercise caution in cases with peripheral anterior synechiae after DMEK. Long-term steroid administration is necessary to prevent graft rejection.

\section{Introduction}

Four types of corneal transplantation are performed: penetrating keratoplasty (PK) [1], in which the entire corneal layer is transplanted due to irreversible corneal opacity of all layers; anterior lamellar keratoplasty, which is localized to the anterior cornea, i.e., only the anterior section is transplanted for patients with corneal opacity; Descemet's stripping automated endothelial keratoplasty (DSAEK), in which part of the stromal and endothelial cell layers are transplanted [2]; and Descemet's membrane endothelial keratoplasty (DMEK) for corneal endothelial damage [3]. DMEK involves transplantation of only the corneal endothelial cell layer, which is approximately $20-\mu \mathrm{m}$ thick, and is more clinically effective than DSAEK [4]. In DMEK, the corneal graft includes only the Descemet's membrane and corneal endothelium, which demonstrates low antigenicity when compared with the stroma and epithelium [5]. Therefore, DMEK has been reported to have a lower rejection rate than other types of corneal transplantation, including PK and DSAEK [6]. Postoperative rejection rates are reported to range between $4.9 \%$ and $28.9 \%$ for PK, $1.6 \%$ and $8.5 \%$ for DSAEK, and $0 \%$ and $2.4 \%$ for DMEK $[7,8]$. Peripheral anterior synechiae are one of the risk factors for graft rejection after PK [9]; however, only a few studies have reported graft failure after DMEK with anterior synechia [10]. Herein, we report a case of graft rejection after DMEK for bullous keratopathy induced by laser iridotomy (LI) where peripheral anterior synechiae were observed postoperatively.

\section{Case Report}

A 66-year-old woman was referred to our hospital with complaints of decreased visual acuity of her right eye. She had undergone bilateral LI for primary angle closure 3 years before and was experiencing bullous keratopathy. At the first visit, her right cornea was edematous with folds of Descemet's membrane and a central corneal thickness (CCT) of $702 \mu \mathrm{m}$. The anterior chambers were shallow, with a depth of $2.36 \mathrm{~mm}$ in the right eye and $2.23 \mathrm{~mm}$ in the left eye. The LI hole was open without intraocular inflammation or hyperemia. Her bestcorrected visual acuity (BCVA) was 20/40. The corneal endothelial cell density (ECD) was unmeasurable in the right eye owing to edematous cornea; in contrast, it was 1,773 cells $/ \mathrm{mm}^{2}$ in the left eye.

Phacoemulsification and intraocular lens implantation were performed in her right eye. Her BCVA was 20/50 1 week after the operation and 20/125 at 2 months after surgery. Since there was no improvement in her BCVA after phacoemulsification and intraocular lens implantation, it was decided that the patient would undergo DMEK. DMEK was performed without further difficulty, and the graft was completely attached with improved transparency, except for peripheral anterior synechiae, which had progressed postoperatively at the temporal cornea (shown in Fig. 1a, b). After 1 month, her BCVA recovered to 20/20, ECD was 1,984 cells $/ \mathrm{mm}^{2}$, and CCT was $477 \mu \mathrm{m}$. Peripheral anterior synechiae at the temporal cornea were also observed postoperatively. Perioperative oral prednisolone administration was stopped 1 month postoperatively, and only $0.1 \%$ topical betamethasone instillation was continued, 


\section{Case Reports in Ophthalmology}

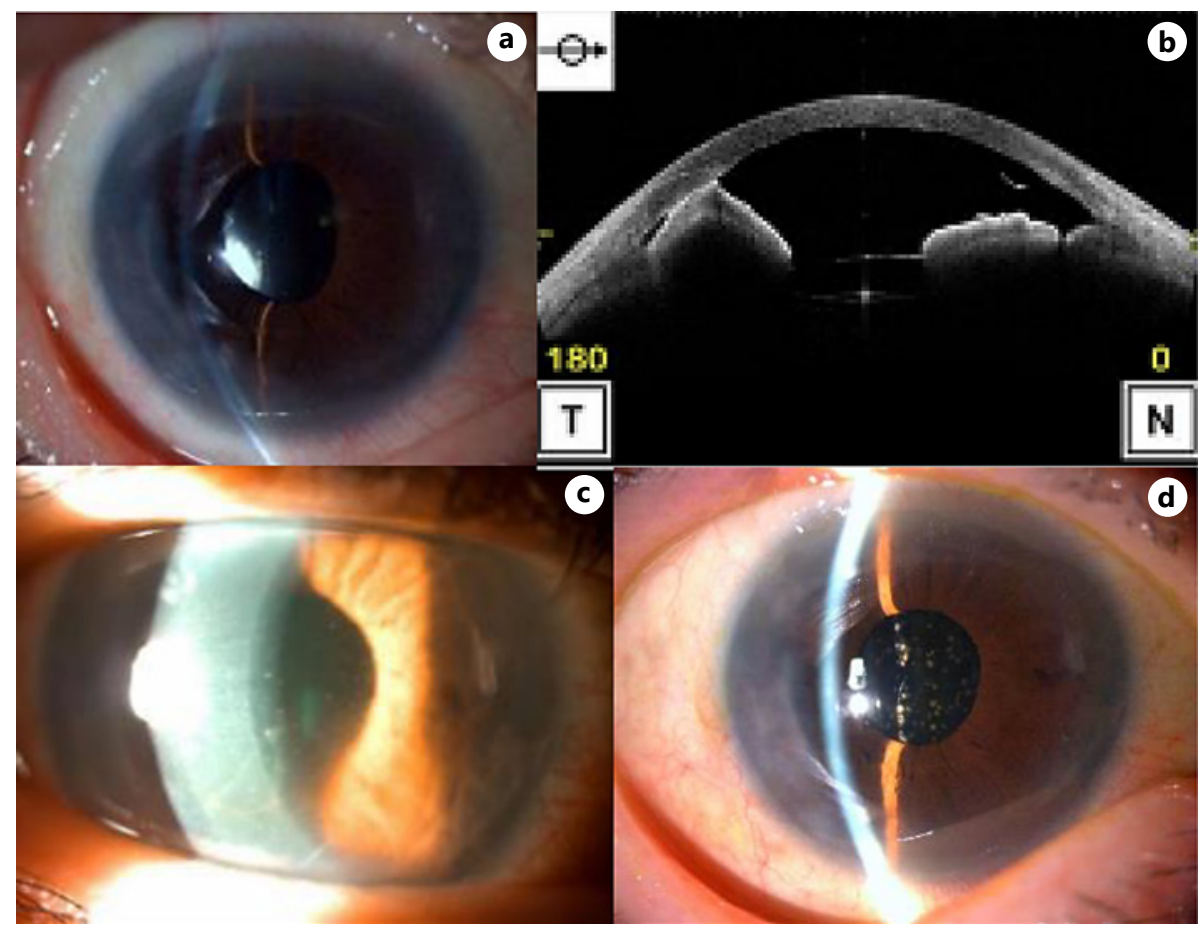

Fig. 1. Clinical images of the anterior segment of the right eye of the patient. a Slit-lamp examination showing a completely attached corneal graft with good transparency after DMEK. b Anterior segment optical coherence tomography image showing peripheral anterior synechiae at the temporal cornea. c Slit-lamp examination showing corneal edema of the donor and graft with intraocular inflammation. $\mathbf{d}$ After 3 subconjunctival injections of betamethasone, there was recovery of DMEK graft rejection, and good transparency was observed. DMEK, Descemet's membrane endothelial keratoplasty.

which was safely and gradually tapered from 4 times to once a day when corneal transparency fully recovered with a BCVA of 20/20, CCT of $486 \mu \mathrm{m}$, and ECD of 1,114 cells $/ \mathrm{mm}^{2}$.

When the dosage of topical instillation was changed to $0.1 \%$ fluorometholone once daily 15 months after DMEK, corneal edema appeared with hyperemia, many Descemet's membrane folds, mutton fat keratic precipitates (KPs) that were diffuse and inferiorly located on the graft, and cells in the anterior chamber (shown in Fig. 1c). Her BCVA worsened to 20/32, and CCT was $704 \mu \mathrm{m}$. Her ECD, which was 980 cells $/ \mathrm{mm}^{2} 2$ months earlier, became unmeasurable. Graft rejection after corneal transplantation or corneal endotheliitis was suspected. Anterior segment optical coherence tomography disclosed that peripheral anterior synechiae were observed only in the recipient cornea. The patient did not wish to undergo a polymerase chain reaction (PCR) test to examine the causative agent. Blood tests did not reveal elevation of antibodies against herpes simplex virus or varicella zoster virus. Therefore, graft rejection was diagnosed, and a subconjunctival injection of dexamethasone was administered 3 times once every 2-3 days and betamethasone topically 6 times a day.

The hyperemia, mutton fat KPs, and cells in the anterior chamber disappeared, and the patient's BCVA recovered to 20/20 2 weeks after the occurrence of the rejection. Topical betamethasone administration was reduced to 4 times a day (shown in Fig. 1d). Two months after graft rejection, administration of $0.1 \%$ betamethasone was further reduced to twice daily, and the ECD reduced to 751 cells $/ \mathrm{mm}^{2}$. Ten months after graft rejection, there was no recurrence of intraocular inflammation with administration of $0.1 \%$ betamethasone twice daily, although CCT was $497 \mu \mathrm{m}$, ECD was 475 cells $/ \mathrm{mm}^{2}$, and BCVA was 20/16. 


\section{Case Reports in Ophthalmology}

\section{Discussion}

This manuscript describes a case of allograft rejection with peripheral anterior synechiae following DMEK. The reactivity to topical steroids and the distribution of KPs indicated that the case was not of corneal endotheliitis but of corneal graft rejection instead. In this case, all the characteristic findings of rejection, such as KPs, intraocular cells, corneal edema, hyperemia, and endothelial precipitates, were present [11]. In addition, endothelial cells typically become irregular in shape, size, and distribution several months before the onset of rejection [12]. Our case also demonstrates the relatively rapid changes in corneal endothelial cells during rejection, such as the reduction of ECD from 1,984 to 1,114 cells $/ \mathrm{mm}^{2} 1$ year before graft rejection and to 980 cells $/ \mathrm{mm}^{2} 2$ months before graft rejection.

Corneal endotheliitis caused by herpes simplex virus or cytomegalovirus is a major consideration during differential diagnosis of graft rejection because it has similar clinical findings. Herpes simplex virus infection is characterized by discoidal KPs, while cytomegalovirus infection is characterized by coin-shaped KPs [11]. In this case, the diagnosis of graft rejection was based on diffuse mutton fat KPs, good response to steroids, and decrease in endothelial cells. Intraocular pressure elevation sometimes observed in patients with corneal endotheliitis owing to viral infection was not detected in the present case. PCR testing should be performed to rule out infection if the treatment has no effect on the inflammation.

Corneal graft rejection after DMEK is rare, and a rate of $1 \%$ was reported at 2 years $[7,13]$. However, discontinuation of postoperative immunosuppressive agents, such as topical steroids, could lead to graft rejection in an estimated $6 \%$ of patients [8]. Moreover, graft rejection often occurs during topical $0.1 \%$ fluorometholone instillation [14]. In this case, graft rejection occurred when topical steroid instillation was reduced in the second postoperative year. The details of the inducible mechanism of rejection are unknown, but anterior synechiae, involving attachment of the recipient's iris and the donor's corneal endothelium, could be a risk factor. In a study using a murine corneal transplant model comparing groups with and without anterior synechiae, the group with anterior synechiae was significantly more likely to undergo rejection [15]. This rejection may occur because anterior synechiae can induce cytotoxic T-lymphocyte activity [15]. In our case, peripheral anterior synechiae developed following DMEK, suggesting that the patient may have been prone to rejection.

This report has a few limitations. First, the differential diagnosis was incomplete, as the patient was unwilling to undergo PCR testing. Second, the time course changes in the shape and degree of the anterior synechia were not fully analyzed. Further basic and clinical research is necessary to elucidate how corneal rejection is associated with the contact between the iris and the corneal endothelium.

In conclusion, prolonged postoperative administration of topical steroids is necessary to prevent graft rejection. Moreover, it is important to recognize peripheral anterior synechiae as a risk factor for rejection during slit-lamp examination for DMEK patients.

\section{Statement of Ethics}

The protocol for this retrospective study was approved and the need for written informed consent was waived on condition of providing the patient with the opportunity to opt out by the Institutional Review Board and Ethics Committee of the University of Tokyo Graduate School of Medicine (Approval No. NI20200006). All clinical procedures adhered to the tenets of the Declaration of Helsinki. Written informed consent was obtained from the patient for publication of this case report and any accompanying images.

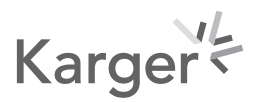




\section{Case Reports in Ophthalmology}

\begin{tabular}{|c|c|}
\hline \multicolumn{2}{|c|}{ Case Rep Ophthalmol 2022;13:17-22 } \\
\hline DOI: $10.1159 / 000520877$ & $\begin{array}{l}\text { (c) } 2022 \text { The Author(s). Published by S. Karger AG, Basel } \\
\text { www.karger.com/cop }\end{array}$ \\
\hline
\end{tabular}

Miyoshi et al.: Corneal Graft Rejection after Descemet's Membrane Endothelial Keratoplasty with Peripheral Anterior Synechiae

\section{Conflict of Interest Statement}

The authors have no conflicts of interest to declare.

\section{Funding Sources}

The research did not receive any grant from funding agencies in the public, commercial, or not-for-profit sectors.

\section{Author Contributions}

Y.M., T.O., S.S., and T.M. contributed to the design and conduct of the study. T.O., Y.M., and S.S. collected, managed, analyzed, and interpreted the data. T.O., Y.M., T.M., T.U., and T.H. prepared and reviewed the final manuscript. All authors have read and approved the final version of this manuscript.

\section{Data Availability Statement}

The data that support the findings of this study are available on request from the corresponding author. The data are not publicly available because they contain information that could compromise the privacy of the research participant.

\section{References}

1 Price FW Jr, Whitson WE, Marks RG. Graft survival in four common groups of patients undergoing penetrating keratoplasty. Ophthalmology. 1991 Mar;98(3):322-8.

2 Price MO, Calhoun P, Kollman C, Price FW Jr, Lass JH. Descemet stripping endothelial keratoplasty: ten-year endothelial cell loss compared with penetrating keratoplasty. Ophthalmology. 2016 Jul;123(7):1421-7.

3 Melles GR, Ong TS, Ververs B, van der Wees J. Descemet membrane endothelial keratoplasty (DMEK). Cornea. 2006 Sep;25(8):987-90.

4 Ham L, Dapena I, Liarakos VS, Baydoun L, van Dijk K, Ilyas A, et al., Midterm results of descemet membrane endothelial keratoplasty: 4 to 7 years clinical outcome. Am J Ophthalmol. 2016 Nov;171:113-21.

5 Hori J, Joyce NC, Streilein JW. Immune privilege and immunogenicity reside among different layers of the mouse cornea. Invest Ophthalmol Vis Sci. 2000 Sep;41(10):3032-42.

6 Stuart AJ, Romano V, Virgili G, Shortt AJ. Descemet's membrane endothelial keratoplasty (DMEK) versus descemet's stripping automated endothelial keratoplasty (DSAEK) for corneal endothelial failure. Cochrane Database Syst Rev. 2018;6(6):CD012097.

7 Pavlovic I, Shajari M, Herrmann E, Schmack I, Lencova A, Kohnen T. Meta-analysis of postoperative outcome parameters comparing descemet membrane endothelial keratoplasty versus descemet stripping automated endothelial keratoplasty. Cornea. 2017 Dec;36(12):1445-51.

8 Price MO, Scanameo A, Feng MT, Price FW Jr. Descemet's membrane endothelial keratoplasty: Risk of immunologic rejection episodes after discontinuing topical corticosteroids. Ophthalmology. 2016 Jun;123(6): 1232-6.

9 Price MO, Thompson RW Jr, Price FW Jr. Risk factors for various causes of failure in initial corneal grafts. Arch Ophthalmol. 2003 Aug;121(8):1087-92.

10 Carreras-Castañer X, Batlle-Ferrando S, Marin-Martinez S, Miguel L, Feu-Basilio S, Peraza-Nieves JE. Anterior synechiae related to an incipient descemet's membrane endothelial keratoplasty (DMEK) allograft rejection. J Fr Ophtalmol. 2020 Dec;43(10):e389-91.

11 Moshirfar M, Murri MS, Shah TJ, Skanchy DF, Tuckfield JQ, Ronquillo YC, et al., A review of corneal endotheliitis and endotheliopathy: differential diagnosis, evaluation, and treatment. Ophthalmol Ther. 2019 Jun;8(2):195-213.

12 Hos D, Matthaei M, Bock F, Maruyama K, Notara M, Clahsen T, et al., Immune reactions after modern lamellar (DALK, DSAEK, DMEK) versus conventional penetrating corneal transplantation. Prog Retin Eye Res. 2019 Nov; 73:100768. 
13 Anshu A, Price MO, Price FW Jr. Risk of corneal transplant rejection significantly reduced with descemet's membrane endothelial keratoplasty. Ophthalmology. 2012 Mar;119(3):536-40.

14 Baydoun L, Livny E, Ham L, Bruinsma M, Melles GRJ. 360-degree Scheimpflug imaging to predict allograft rejection after descemet membrane endothelial keratoplasty. Cornea. 2016 Nov;35(11):1385-90.

15 Yamagami S, Tsuru T. Increase in orthotopic murine corneal transplantation rejection rate with anterior synechiae. Invest Ophthalmol Vis Sci. 1999 Sep;40(10):2422-6. 